The Astrophysical Journal, 277:576-580, 1984 February 15

(c) 1984. The American Astronomical Society. All rights reserved. Printed in U.S.A.

\title{
PHOTODISSOCIATION OF OH IN INTERSTELLAR CLOUDS
}

\author{
Ewine F. van Dishoeck ${ }^{1,2}$ AND A. Dalgarno ${ }^{1}$ \\ Received 1983 June 13; accepted 1983 July 28
}

\begin{abstract}
Calculations are presented of the lifetime of $\mathrm{OH}$ against photodissociation by the interstellar radiation field as a function of depth into interstellar clouds containing grains of various scattering properties. The effectiveness of the different photodissociation channels changes with depth into a cloud. Absorption into the repulsive $1^{2} \Sigma^{-}$ state is usually the main dissociation channel, but with forward-scattering grains in clouds of small optical depths, absorption into the $3^{2} \Pi$ state is more effective.
\end{abstract}

Subject headings: interstellar: molecules - molecular processes

\section{INTRODUCTION}

The hydroxyl radical, $\mathrm{OH}$, occurs in diffuse and dense interstellar clouds and in shocked and maser regions of the interstellar gas. The most uncertain element in chemical schemes proposed for the formation and destruction of $\mathrm{OH}$ is the efficiency with which it undergoes photodissociation. Recently, detailed studies have been carried out (van Dishoeck, Langhoff, and Dalgarno 1983; van Dishoeck and Dalgarno 1983; van Dishoeck et al. 1983) of the dissociation channels of $\mathrm{OH}$, and the cross sections effective in dissociating $\mathrm{OH}$ have been calculated. We use them here to calculate the destruction rates of $\mathrm{OH}$ in interstellar clouds. The rates depend upon the scattering properties of the grains, and we present results for three different choices of grain albedo and scattering anisotropy.

\section{PHOTODISSOCIATION CHANNELS}

In its ground electronic state, $\mathrm{OH}$ has ${ }^{2} \Pi$ symmetry. Allowed electric dipole absorptions occur into excited ${ }^{2} \Sigma^{+},{ }^{2} \Sigma^{-},{ }^{2} \Pi$, and ${ }^{2} \Delta$ states. The potential energy curves are illustrated in Figure 1. Direct photodissociation occurs following absorption into the repulsive $1^{2} \Sigma^{-}, 1^{2} \Delta$, and $B^{2} \Sigma^{+}$states. Predissociation occurs with unit efficiency following absorption into vibrational levels of the $A^{2} \Sigma^{+}$state with quantum numbers greater than one. Absorption into the discrete levels of the $D^{2} \Sigma^{-}$state may be followed by spontaneous radiative decay into the lower lying $1^{2} \Sigma^{-}$state, which then dissociates, or into the discrete levels of the ground state. The $3{ }^{2} \Pi$ state vibrational levels undergo predissociation by radial coupling to the repulsive $2^{2} \Pi$ state. A complex pattern of responses occurs for the other excited states which are accessible to photons with energies below the Lyman limit.

Table 1 contains a list of excited states of $\mathrm{OH}$ which are populated by discrete line absorptions from the $X^{2} \Pi$ state by photons with wavelengths larger than $912 \AA$. The corresponding band oscillator strengths $f_{0 v^{\prime}}$ for transitions from the $v^{\prime \prime}=0$ vibrational level of the $X^{2} \Pi$ state to the $v^{\prime}$ vibrational levels of the excited states and the probabilities $\eta_{v^{\prime}}$ that population of the $v^{\prime}$ level is followed by dissociation have been

\footnotetext{
${ }^{1}$ Harvard-Smithsonian Center for Astrophysics, Cambridge, Mass.

${ }^{2}$ Sterrewacht, Leiden, The Netherlands.
}

calculated by van Dishoeck and Dalgarno (1983). The dissociation efficiency of any particular channel may be characterized by an effective cross section integrated over wavelength such that

$$
\Delta=\int \sigma(\lambda) d \lambda=8.85 \times 10^{-21} \sum_{v^{\prime}} \lambda_{0 v^{\prime}}^{2} f_{0 v^{\prime}} \eta_{v^{\prime}} \mathrm{cm}^{2} \AA,
$$

where $\lambda_{0 v^{\prime}}$ is the absorption wavelength in $\AA$. Values of $\Delta$ and the wavelengths $\lambda_{0 v^{\prime}}$ for $v^{\prime}$ where $f_{0 v^{\prime}}$ is a maximum for a given

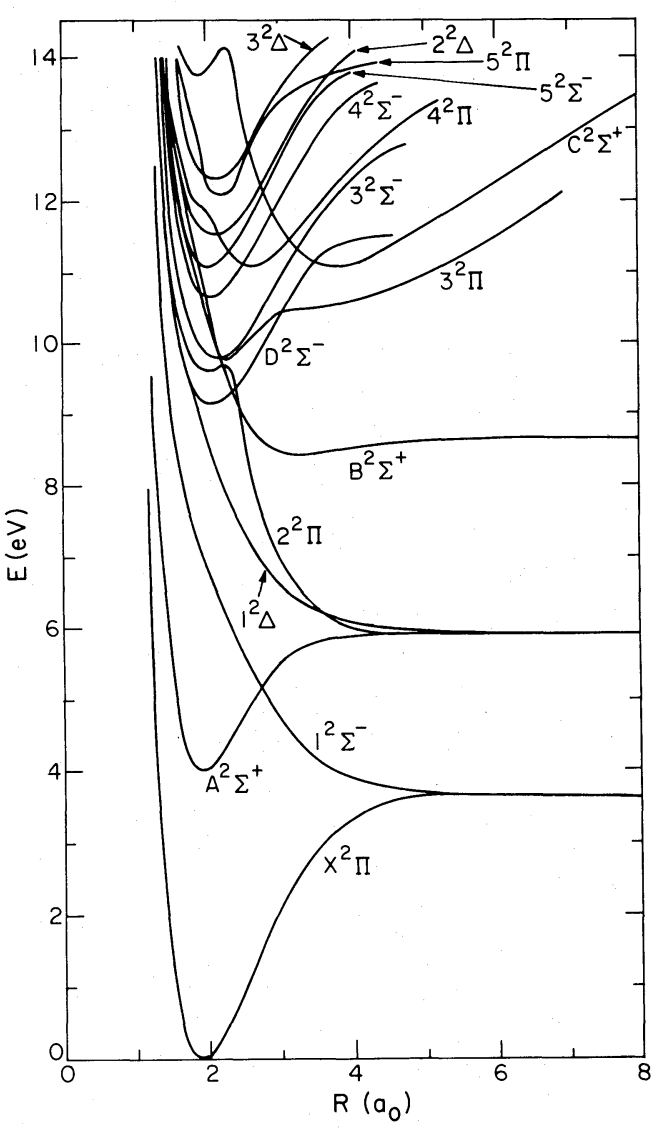

Fig. 1.-Potential energy curves for $\mathrm{OH}$ 
TABLE 1

Effective Photodissociation Cross Sections $\Delta$ and EFfective Absorption Wavelengths $\lambda_{0 v^{\prime}}$ FOR Discrete TRansitions in OH

\begin{tabular}{|c|c|c|}
\hline Electronic State & $\Delta\left(\mathrm{cm}^{2} \AA\right)$ & $\lambda_{0 v^{\prime}}(\AA)$ \\
\hline$A^{2} \Sigma^{+}$ & $3.1(-18)$ & 2618 \\
\hline$D^{2} \Sigma^{-}$ & $3.4(-17)$ & 1220 \\
\hline $3^{2} \Sigma^{-}$ & $8.1(-18)$ & 1095 \\
\hline $4^{2} \Sigma^{-}$ & $1.2(-18)$ & 1040 \\
\hline $5^{2} \Sigma^{-}$ & $1.1(-17)$ & 1000 \\
\hline $4^{2} \Pi$ & $1.7(-17)$ & 1020 \\
\hline $5^{2} \Pi$ & $1.1(-16)$ & 1000 \\
\hline $2^{2} \Delta$. & $2.0(-17)$ & 1040 \\
\hline $3^{2} \Delta$. & $2.2(-18)$ & 1010 \\
\hline
\end{tabular}

electronic transition are included in Table 1. The accuracy of the calculations is uncertain for the higher lying states.

The cross sections adopted for the direct photodissociation channels are collected in Figure 2 (van Dishoeck, Langhoff, and Dalgarno 1983; van Dishoeck and Dalgarno 1983). The magnitudes of the cross sections are probably accurate to better than $20 \%$ and the positions of the maxima to better than $\pm 30 \AA$. Included in Figure 2 is the smooth background cross section resulting from absorption into the coupled $2{ }^{2} \Pi$ and $3{ }^{2} \Pi$ states (van Dishoeck et al. 1983). We shall refer to both these states as the $3^{2} \Pi$ channel. The effective cross section for the $3{ }^{2} \Pi$ channel is about $15 \%$ larger than the value adopted by van Dishoeck and Dalgarno (1983) for discrete absorptions into the $3{ }^{2} \Pi$ state. The $3{ }^{2} \Pi$ channel dominates the photodissociation at shorter wavelengths. At longer wavelengths direct photodissociation is due mainly to the $1^{2} \Sigma^{-}$channel. The total cross section at the Lyman-alpha wavelength of $1216 \AA$ is $1.8 \times 10^{-18} \mathrm{~cm}^{2}$, a value which is uncertain by about $30 \%$ because of possible errors in the calculated excitation energies of the states.

\section{PHOTODISSOCIATION RATES}

The photodissociation rates, $\Gamma$, of molecules subjected to the interstellar radiation field depend upon the extinction, the albedo, and the scattering phase function of the interstellar grains (Sandell and Mattila 1975; Whitworth 1975; Bernes and Sandqvist 1977; Roberge, Dalgarno, and Flannery 1981), none of which is known accurately. We adopted the three models used by Roberge et al., which are specified by parameters $x_{\lambda}$, $\omega_{\lambda}$, and $g_{\lambda}$. The parameter $x_{\lambda}=\tau_{\lambda} / \tau_{v}$, where $\tau_{\lambda}$ is the optical depth for dust extinction at wavelength $\lambda$, and $\tau_{v}$ is the optical depth in the visual band. The parameter $\omega_{\lambda}$ is the albedo at $\lambda$, and $g_{\lambda}$ is an asymmetry measure which varies from zero for isotropic scattering to unity for scattering that is entirely in the forward direction. The dependencies on wavelength of $x_{\lambda}, \omega_{\lambda}$, and $g_{\lambda}$ are presented by Roberge et al., who also recommended values for $I_{\lambda}^{0}$, the unattenuated interstellar radiation field. Models (2) and (3) have large values of $\omega_{\lambda}$ and $g_{\lambda}$, especially for $\lambda<1550 \AA$, whereas in model (1) the scattering is isotropic at ultraviolet wavelengths.

The radiative transfer equations were solved by the method of Flannery, Roberge, and Rybicki (1980) for several semiinfinite plane-parallel clouds of uniform density with total visual extinctions $A_{v}$ ranging from 1 to $100 \mathrm{mag}$ illuminated isotropically on both faces by a radiation field with intensity $I_{\lambda}^{0}$.

Figures 3 and 4 present the contributions of the individual photodissociation channels at different depths into clouds with a visual extinction of 1 and 10 mag for the three grain models of Roberge et al. Throughout the clouds continuum absorptions into the repulsive $1^{2} \Sigma^{-}, 1^{2} \Delta$, and $3^{2} \Pi$ channels provide the most efficient paths for dissociation though photodissociation initiated by line absorptions into the $D^{2} \Sigma^{-}$and $5^{2} \Pi$ states is significant at small optical depths. The $A^{2} \Sigma^{+}$ state is of minor importance, although it is the longest wavelength channel. The contributions from the high-lying states diminish more rapidly with depth into the cloud, especially for models (1) and (2), because of the increased dust extinction at shorter wavelengths.

Photodissociation by absorption into the $1^{2} \Sigma^{-}$state leads to ground-state $\mathrm{O}\left({ }^{3} \mathrm{P}\right)$ and $\mathrm{H}$ atoms, and absorptions into the $1{ }^{2} \Delta$ and $3{ }^{2} \Pi$ states lead to excited $\mathrm{O}\left({ }^{1} D\right)$ and $\mathrm{H}$ atoms. For small optical depths in models (1) and (2) and for the forward-

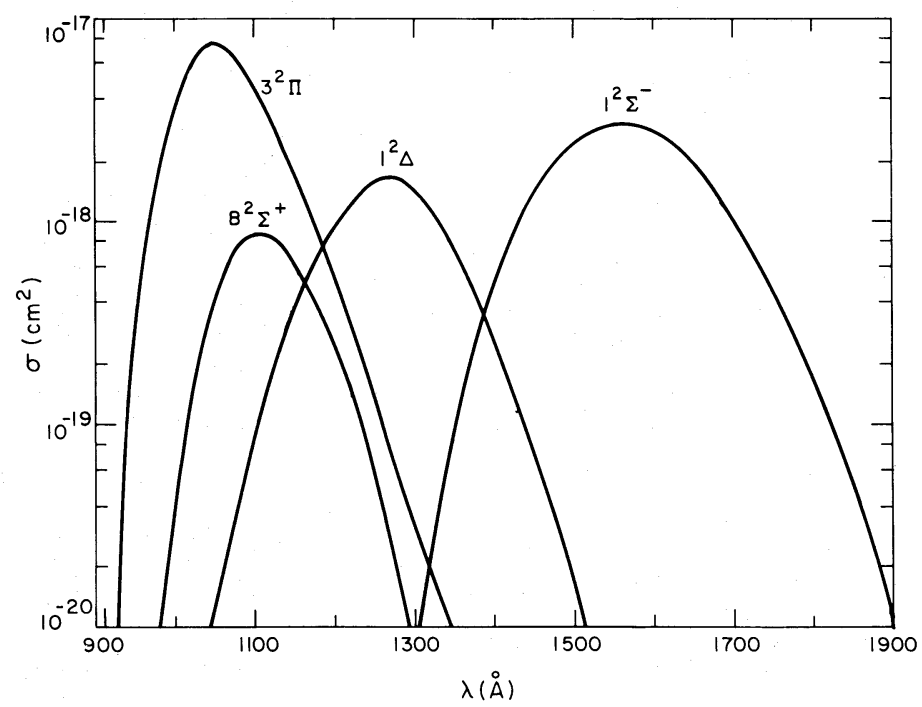

FIG. 2.-Photodissociation cross sections for $\mathrm{OH}$ as a function of wavelength 


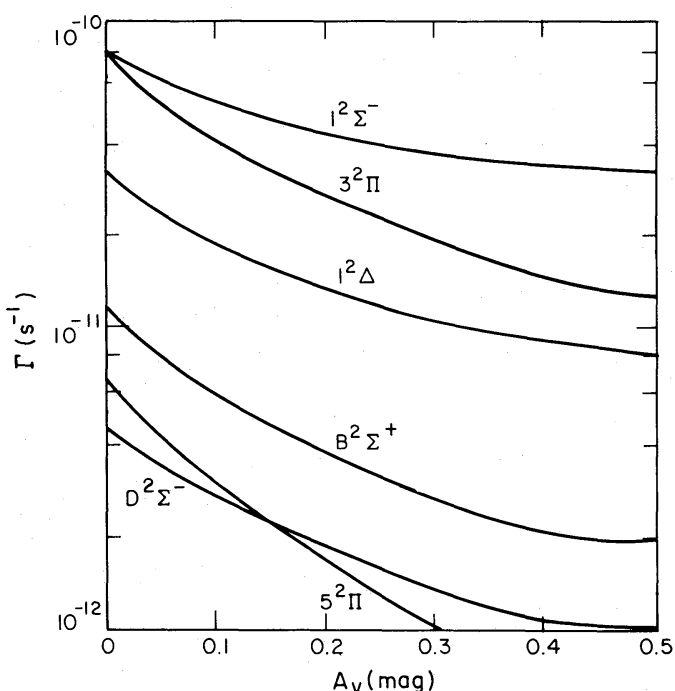

FIG. $3 a$

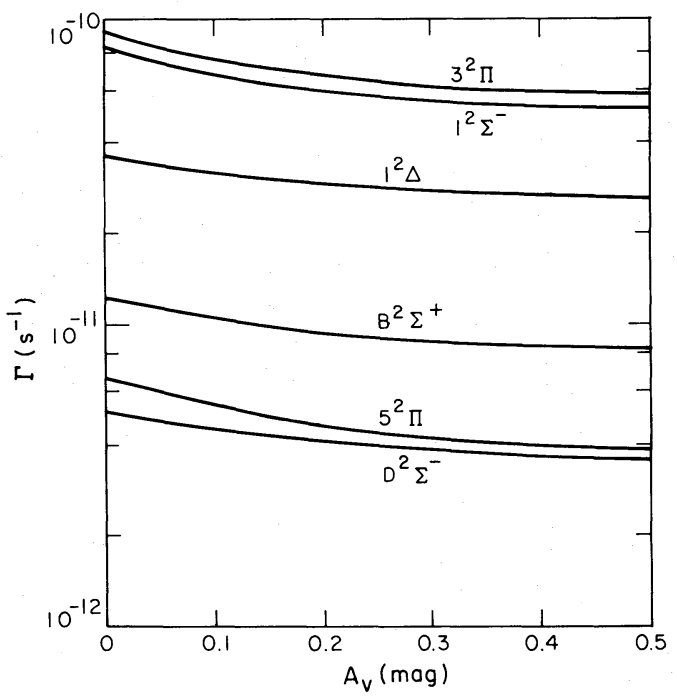

FIG. $3 c$

FIG. 3.-The photodissociation rates for individual channels as a function of optical depth into a cloud of total extinction $A_{v}=1 \mathrm{mag}$ for grain models (a) (1), (b) (2), and (c) (3)

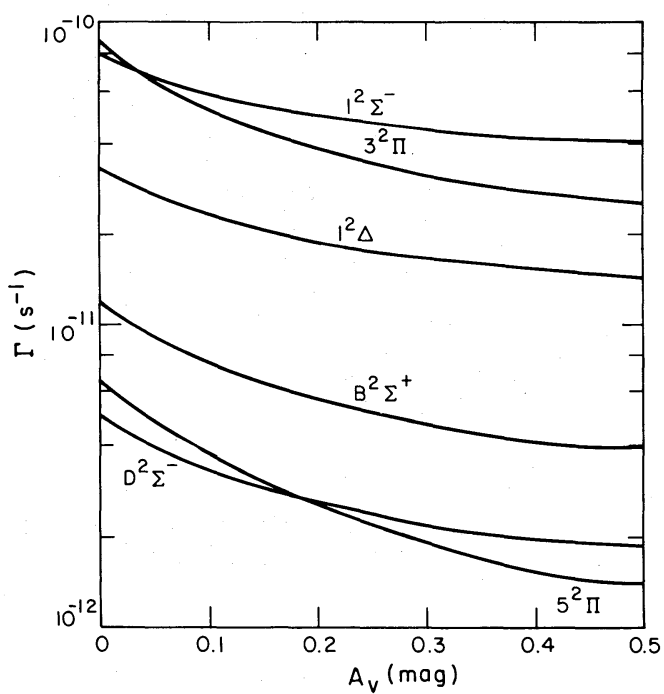

Fig. $3 b$

scattering grain model (3), about equal amounts of $\mathrm{O}\left({ }^{3} P\right)$ and $\mathrm{O}\left({ }^{1} D\right)$ are produced by photodissociation. Deeper into the clouds, relatively more $\mathrm{O}\left({ }^{3} P\right)$ will be produced, because of the decreased contribution from the higher lying $3{ }^{2} \Pi$ channel.

In previous estimates of the photodissociation rate of $\mathrm{OH}$ it was assumed that the photodissociation is dominated by absorptions into the $C^{2} \Sigma^{+}$state at $950 \AA$, followed by predissociation (Smith and Stella 1975; Sandell 1978; Roberge, Dalgarno, and Flannery 1981). Because of the short wavelength of the transition, the corresponding photodissociation rate diminishes rapidly with increasing optical depth. It is further reduced by the opacity due to molecular hydrogen and carbon. However, as discussed by van Dishoeck and Dalgarno (1983), the $C^{2} \Sigma^{+}$channel contributes negligibly to the photodissociation. The important $1^{2} \Sigma^{-}$channel lies at much longer wavelengths and is not modified by the shielding by hydrogen and carbon. The $1^{2} \Sigma^{-}$channel was considered before (Sandell 1978), but its effective cross section was underestimated by a factor of 40 . The new photodissociation rates are larger than the previous ones by a factor which increases from 5 at small optical depths, to between 10 and 100 at larger depths.

The results for a cloud with a total extinction $A_{v}$ of $100 \mathrm{mag}$ are presented in Table 2 for the forward-scattering grain

TABLE 2

Channel Photodissociation Rates $\Gamma\left(\mathrm{s}^{-1}\right)$ for the Forward-Scattering Grain Model (3) in a Cloud with a TOtal ExTinction of 100 MAgNitudes

\begin{tabular}{cccccccc}
\hline \hline Depth $A_{v}(\mathrm{mag})$ & $1^{2} \Sigma^{-}$ & $1^{2} \Delta$ & $B^{2} \Sigma^{+}$ & $3^{2} \Pi$ & $A^{2} \Sigma^{+}$ & $D^{2} \Sigma^{-}$ & $5^{2} \Pi$ \\
\hline $0 \ldots \ldots \ldots \ldots \ldots \ldots \ldots \ldots$ & $6.93(-11)$ & $2.80(-11)$ & $9.49(-12)$ & $7.37(-11)$ & $1.99(-13)$ & $3.35(-12)$ & $5.73(-12)$ \\
$1 \ldots \ldots \ldots \ldots \ldots \ldots \ldots \ldots \ldots$ & $1.35(-11)$ & $8.03(-12)$ & $2.12(-12)$ & $1.59(-11)$ & $2.34(-14)$ & $8.56(-13)$ & $9.24(-13)$ \\
$2 \ldots \ldots \ldots \ldots \ldots \ldots \ldots \ldots$ & $4.68(-12)$ & $3.24(-12)$ & $7.30(-13)$ & $5.45(-12)$ & $4.53(-15)$ & $3.41(-13)$ & $2.44(-13)$ \\
$3 \ldots \ldots \ldots \ldots \ldots \ldots \ldots \ldots$ & $2.01(-12)$ & $1.47(-12)$ & $2.90(-13)$ & $2.06(-12)$ & $9.43(-16)$ & $1.47(-13)$ & $7.48(-14)$ \\
$4 \ldots \ldots \ldots \ldots \ldots \ldots \ldots \ldots \ldots$ & $8.88(-13)$ & $6.88(-13)$ & $1.13(-13)$ & $8.22(-13)$ & $2.03(-16)$ & $6.47(-14)$ & $2.31(-14)$ \\
$5 \ldots \ldots \ldots \ldots \ldots \ldots \ldots \ldots \ldots$ & $4.34(-13)$ & $3.74(-13)$ & $4.60(-14)$ & $3.41(-13)$ & $4.41(-17)$ & $3.00(-14)$ & $7.81(-15)$ \\
$6 \ldots \ldots \ldots \ldots \ldots \ldots \ldots \ldots \ldots$ & $2.22(-13)$ & $1.67(-13)$ & $2.05(-14)$ & $1.46(-13)$ & $9.64(-18)$ & $1.40(-14)$ & $2.56(-15)$ \\
$8 \ldots \ldots \ldots \ldots \ldots \ldots \ldots \ldots$. & $6.25(-14)$ & $4.37(-14)$ & $4.05(-15)$ & $2.82(-14)$ & $4.66(-19)$ & $3.22(-15)$ & $3.05(-16)$ \\
$10 \ldots \ldots \ldots \ldots \ldots \ldots \ldots \ldots$ & $1.88(-14)$ & $1.20(-14)$ & $8.43(-16)$ & $5.80(-15)$ & $2.26(-20)$ & $7.73(-16)$ & $3.79(-17)$ \\
$15 \ldots \ldots \ldots \ldots \ldots \ldots \ldots \ldots$ & $1.05(-15)$ & $6.98(-16)$ & $2.40(-17)$ & $1.34(-16)$ & $1.18(-23)$ & $2.18(-17)$ & $2.39(-19)$ \\
$20 \ldots \ldots \ldots \ldots \ldots \ldots \ldots \ldots$ & $6.67(-17)$ & $3.74(-17)$ & $7.57(-19)$ & $3.82(-18)$ & $6.21(-27)$ & $7.20(-19)$ & $1.64(-21)$ \\
$25 \ldots \ldots \ldots \ldots \ldots \ldots \ldots \ldots$ & $4.54(-18)$ & $2.17(-18)$ & $2.75(-20)$ & $1.29(-19)$ & $3.26(-30)$ & $2.54(-20)$ & $1.23(-23)$ \\
\hline
\end{tabular}




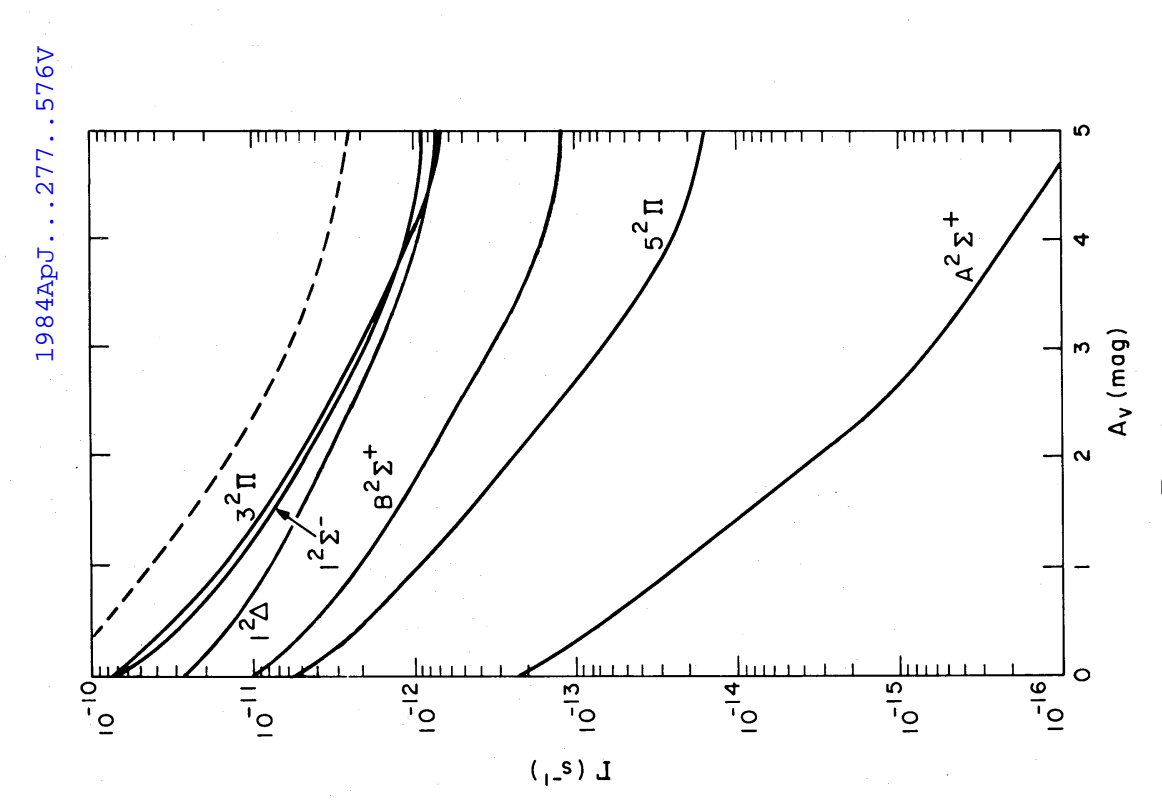

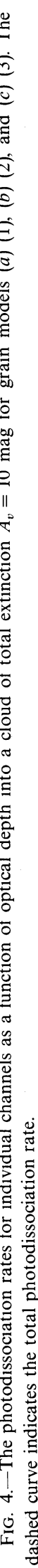

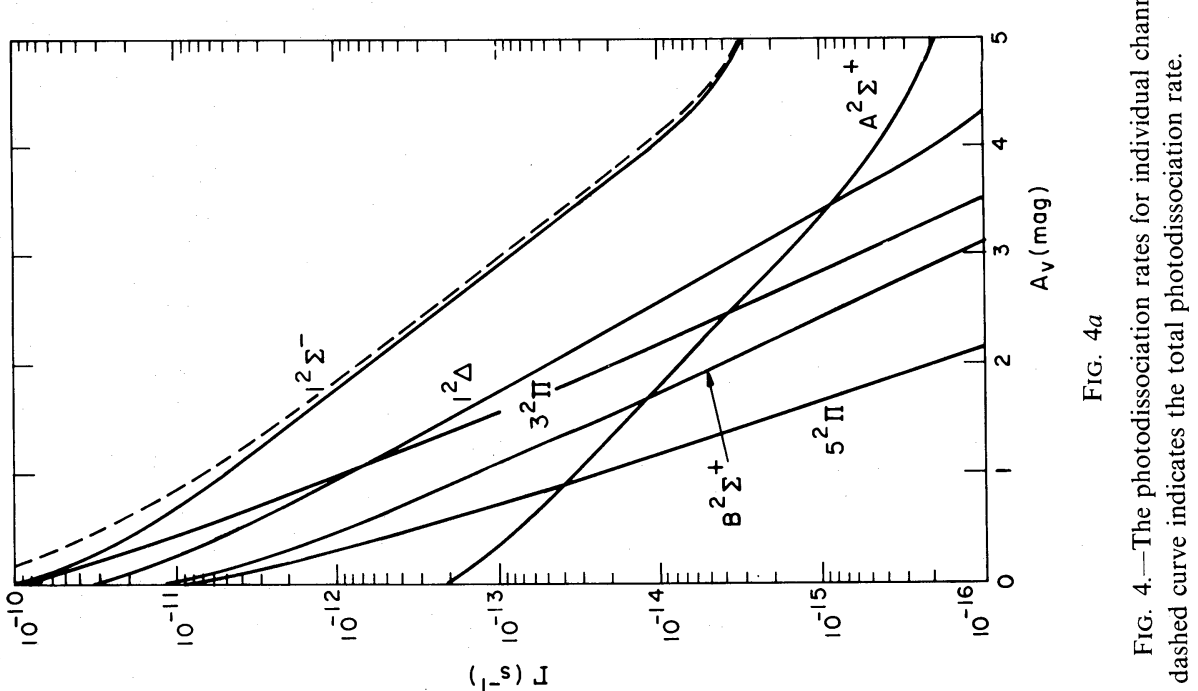




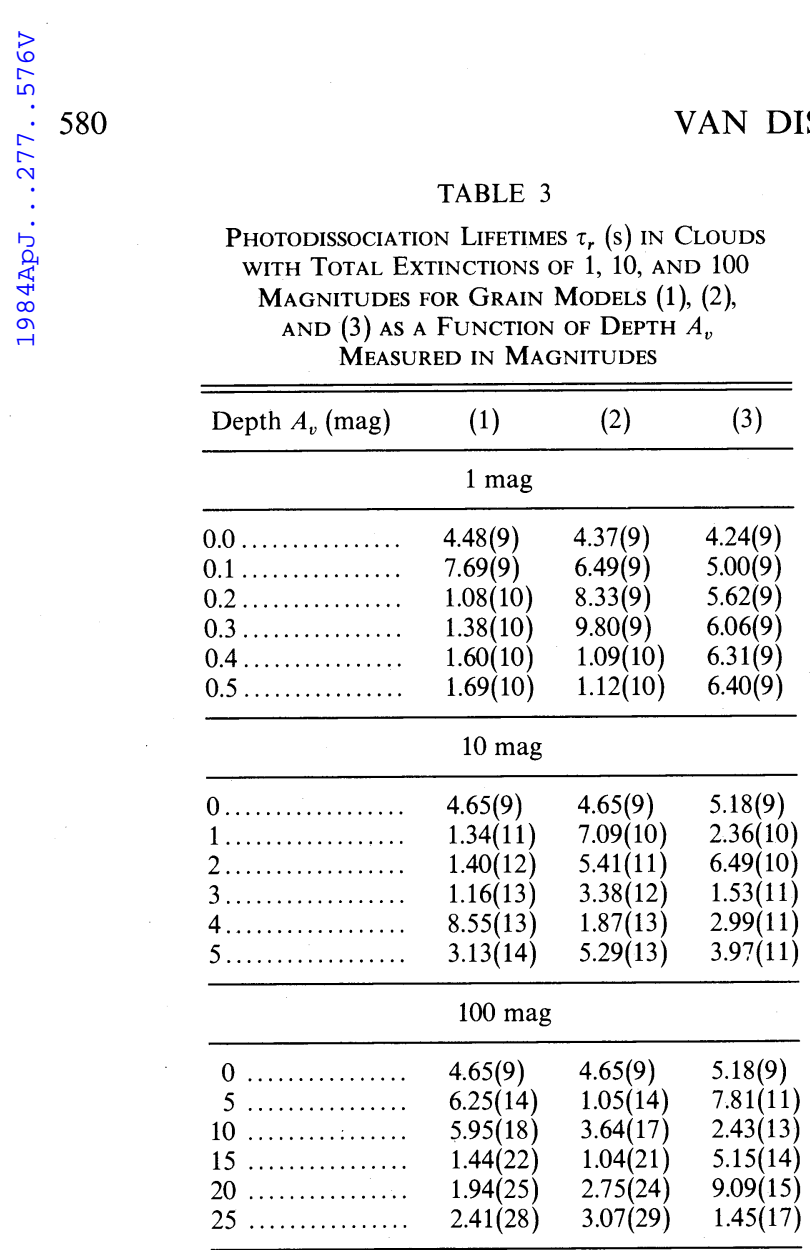

model (3). The anisotropy exerts a substantial influence on the rates of photodissociation deep into the cloud. For depths corresponding to $A_{v}<50 \mathrm{mag}$, the results are also appropriate to a cloud illuminated on one side only.

The calculated lifetimes $\tau_{r}$ of $\mathrm{OH}$ against photodissociation for the three grain models in clouds of total extinctions 1,10 , and $100 \mathrm{mag}$ are given in Table 3 . The corresponding total photodissociation rates may be represented by the formula

$$
\Gamma=c \exp \left(-\alpha A_{v}-\beta A_{v}^{2}\right) \mathrm{s}^{-1},
$$

to within a standard deviation of less than $10 \%$ for $A_{v} \leq 10 \mathrm{mag}$. The formula should be useful in the construction of models of molecular clouds. The constants $c, \alpha$, and $\beta$ for several clouds of total extinctions between 1 and 30 mag are given in Table 4. For diffuse clouds with $A_{v} \lesssim 3$, the rate varies slowly with optical depth, and photodissociation is the main destruction mechanism throughout. For denser clouds, the destruction of $\mathrm{OH}$ by photodissociation competes with that by chemical reactions of $\mathrm{OH}$ with ions such as $\mathrm{H}^{+}, \mathrm{H}_{3}^{+}, \mathrm{C}^{+}, \mathrm{Si}^{+}$, and $\mathrm{HCO}^{+}$and with neutral species such as $\mathrm{C}, \mathrm{O}$, and $\mathrm{CO}$. For forward-scattering grains, photodissociation is significant into large optical depths.

We are greatly indebted to Dr. W. G. Roberge for making available his program for the solution of the equations of radiative transfer and to Dr. J. H. Black for assisting us in its use. This research was partly supported by the Netherlands Organization for the Advancement of Pure Research (ZWO) and partly by the National Science Foundation under grant AST 81-14718.

TABLE 4

Fit of Photodissociation Rates $\Gamma\left(\mathrm{s}^{-1}\right)$ to the Formula $c \exp \left(-\alpha A_{v}-\beta A_{v}^{2}\right)$ for Clouds with Total Extinction RANGing from 1 to 30 Magnitudes for Grain Models (1), (2), AND (3)

\begin{tabular}{|c|c|c|c|c|c|c|c|c|c|}
\hline \multirow{2}{*}{$\begin{array}{l}\text { Total ExTINCTION } \\
\text { (mag) }\end{array}$} & \multicolumn{3}{|c|}{ (1) } & \multicolumn{3}{|c|}{ (2) } & \multicolumn{3}{|c|}{ (3) } \\
\hline & $c$ & $\alpha$ & $\beta$ & $c$ & $\alpha$ & $\beta$ & $c$ & $\alpha$ & $\beta$ \\
\hline & $2.23(-10)$ & 5.327 & -5.500 & $2.29(-10)$ & 3.901 & -4.181 & $2.36(-10)$ & 1.735 & -1.868 \\
\hline & $2.16(-10)$ & 5.086 & -2.149 & $2.20(-10)$ & 3.829 & -1.807 & $2.12(-10)$ & 1.898 & -1.023 \\
\hline & $2.15(-10)$ & 4.463 & -1.240 & $2.16(-10)$ & 3.437 & -0.852 & $2.00(-10)$ & 1.947 & -0.697 \\
\hline & $2.15(-10)$ & 4.137 & -0.824 & $2.15(-10)$ & 3.333 & -0.652 & $1.96(-10)$ & 1.827 & -0.463 \\
\hline 5 & $2.15(-10)$ & 3.924 & -0.598 & $2.15(-10)$ & 3.204 & -0.476 & $1.95(-10)$ & 1.768 & -0.344 \\
\hline 6 . & $2.15(-10)$ & 3.678 & -0.439 & $2.15(-10)$ & 3.036 & -0.352 & $1.94(-10)$ & 1.694 & -0.265 \\
\hline 8 & $2.15(-10)$ & 3.309 & -0.263 & $2.15(-10)$ & 2.780 & -0.217 & $1.93(-10)$ & 1.565 & -0.172 \\
\hline 10 & $2.15(-10)$ & 3.137 & -0.189 & $2.15(-10)$ & 2.651 & -0.158 & $1.93(-10)$ & 1.490 & -0.127 \\
\hline & $2.15(-10)$ & 2.487 & -0.041 & $2.15(-10)$ & 2.026 & -0.023 & $1.93(-10)$ & 1.083 & -0.024 \\
\hline
\end{tabular}

\section{REFERENCES}

Bernes, C., and Sandqvist, A. 1977, Ap. J., 217, 71.

Flannery, B. P., Roberge, W. G., and Rybicki, G. 1980, Ap. J., 236, 598

Roberge, W. G., Dalgarno, A., and Flannery, B. P. 1981, Ap. J., 243, 817.

Sandell, G. 1978, Astr. Ap., 69, 85 .

Sandell, G., and Mattila, K. 1975, Astr. Ap., 42, 357.

Smith, W. H., and Stella, G. 1975, J. Chem. Phys., 63, 2395. van Dishoeck, E. F., and Dalgarno, A. 1983, J. Chem. Phys., 79, 873 .

van Dishoeck, E. F., Langhoff, S. R., and Dalgarno, A. 1983, J. Chem. Phys., 78, 4552 .

van Dishoeck, E. F., van Hemert, M. C., Allison, A. C., and Dalgarno, A. 1983, in preparation.

Whitworth, A. P. 1975, Ap. Space Sci., 34, 155.

A. Dalgarno: Harvard-Smithsonian Center for Astrophysics, Cambridge, MA 02138

Ewine F. van Dishoeck: Sterrewacht, Huygens Laboratorium, P.O. Box 9513, 2300 RA Leiden, The Netherlands 